\title{
Breaking of forward-backward symmetry in driven systems
}

\author{
Szolnoki, Attila; Szabó, György
}

Published in:

Physical Review E. Statistical, Nonlinear, and Soft Matter Physics

Link to article, DOI:

10.1103/PhysRevE.48.611

Publication date:

1993

Document Version

Publisher's PDF, also known as Version of record

Link back to DTU Orbit

Citation (APA):

Szolnoki, A., \& Szabó, G. (1993). Breaking of forward-backward symmetry in driven systems. Physical Review E. Statistical, Nonlinear, and Soft Matter Physics, 48(1), 611-613. https://doi.org/10.1103/PhysRevE.48.611

\section{General rights}

Copyright and moral rights for the publications made accessible in the public portal are retained by the authors and/or other copyright owners and it is a condition of accessing publications that users recognise and abide by the legal requirements associated with these rights.

- Users may download and print one copy of any publication from the public portal for the purpose of private study or research.

- You may not further distribute the material or use it for any profit-making activity or commercial gain

- You may freely distribute the URL identifying the publication in the public portal

If you believe that this document breaches copyright please contact us providing details, and we will remove access to the work immediately and investigate your claim 


\title{
Breaking of forward-backward symmetry in driven systems
}

\author{
Attila Szolnoki* \\ Department of Physical Chemistry, The Technical University of Denmark, Building 206, DK-2800 Lyngby, Denmark \\ György Szabó \\ KFKI-Research Institute for Materials Science, P. O. Box 49, H-1525 Budapest, Hungary
}

(Received 8 October 1992; revised manuscript received 25 February 1993)

\begin{abstract}
The dynamical pair approximation was modified to study the stationary states in a twodimensional repulsive-lattice-gas model driven far from equilibrium by the application of an external field. This approximation distinguishes between the forward, backward, and transverse directions with respect to the electric field. In the present driven system, the forward-backward symmetry is broken at the level of the pair approximation. The difference between the forward and backward directions is confirmed by Monte Carlo simulations.
\end{abstract}

PACS number(s): 05.50.+q, 64.60.Cn, 66.30.-h

Phase transitions far from equilibrium are extensively studied in very different physical systems. A well known type of nonequilibrium state may be produced by the application of a homogeneous electric field causing persistent particle transport (for a recent review see the paper by Schmittmann [1]). The driven lattice-gas model was first suggested by Katz, Lebowitz, and Spohn [2] as a means of investigating nonequilibrium behavior. They observed that the electric field modifies the particle distribution as well as the ordering transition. This process is accompanied by anisotropic correlations. In the literature $[3-5]$ the transverse and longitudinal directions are distinguished; however, the forward and backward directions are assumed to be equivalent. In fact, the difference between the forward and backward directions has not yet been investigated in these systems except for the onedimensional lattice gas [6]. In this former model, for example, the probabilities of the (0010) and the reflectionsymmetric (0100) configurations at four subsequent sites are not equivalent and the difference is an odd function of the electric field. In this Brief Report we demonstrate that the breaking of forward-backward symmetry may also be observed for a two-dimensional system. For this purpose we analyze the effect of electric field on the particle distribution in a half-filled lattice gas with a repulsive nearest-neighbor interaction. In this model the breaking of the forward-backward symmetry may be observed at the level of pair distribution because the particle distribution is not translation invariant in the ordered phase.

The stochastic dynamics of driven lattice gases is characterized by single-particle jumps to one of the empty neighboring sites (Kawasaki dynamics). The jump rates are biased by a uniform electric field parallel to one of the principal directions $[1,2]$. In other words, the jumps are favored along, and suppressed against the field direction. The difference between the forward and backward jump rates results in modified particle distribution in which the forward-backward (reflection) symmetry is broken. As mentioned above the breaking of the forward-backward symmetry may be observed as a difference between the probabilities of some pairs of $k$-point configurations. In a previous paper [6] we have shown that the probabilities of the (01) and (10) configurations are equivalent if the lattice sites are occupied uniformly. This equivalence is no longer valid if the particle distribution exhibits a long-range order characterized by two sublattices with different occupations. This situation is characteristic of the equilibrium state of a square lattice gas with a repulsive nearest-neighbor interaction.

Adopting the previous notations we choose the particle charge, the coupling, lattice, and Boltzmann constants to be unity. This system exhibits a chessboardlike ordered structure below a critical temperature $T_{c}=0.5673[7]$. In the ordered structure two sublattices ( $A$ and $B$ ) may be distinguished in which the average occupations are different; $n_{A}=1 / 2+x$ and $n_{B}=1 / 2-x$, where $x$ $(|x| \leq 1 / 2)$ is the order parameter. Henceforth we assume that the particles reside dominantly in sublattice $A$, that is, $x>0$.

In lattice-gas models the particle jumps are blocked by the occupancy of the target point. Consequently, the particle fixed at a given point causes congestion in the driven system, i.e., the average occupancy is higher at the preceding point than at the subsequent one. In the ordered structure the particle positions are fixed by the long-range order. This phenomenon led us to distinguish the forward and backward directions when analyzing pair correlations in such systems in which the particles prefer to reside in one of the sublattices.

The present driven lattice-gas model has been studied by Leung, Schmittman, and Zia [4] using renormalization-group technique and by Dickman [5] using dynamical pair approximation, respectively. These authors have shown that the critical temperature decreases with the electric field and the phase transition becomes first order above a threshold value $\left(E_{\mathrm{th}} \approx 1.49\right.$ for the Kawasaki hopping rate). Furthermore, the critical temperature becomes zero at $E=2$ and the ordering 
is completely suppressed at zero temperature if $E>3$ [4].

Our method may be considered as an extension of the dynamical pair approximation used by Dickman for twodimensional driven lattice gases $[5,8]$. We have determined the stationary solution of the equations describing the time evolution of sublattice occupation and probability of $(1,1)$ pairs along the mentioned directions (for details see Refs. $[5,6,8])$. These equations summarize the loss and gain contributions of elementary jumps for all the possible configurations on the sites affecting the jump rates. The probability of the configurations is determined at the level of pair approximation. Instead of the uniform longitudinal pair distribution function, we introduce two quantities: $p_{f}(1,1)$ and $p_{b}(1,1)$, the forward and backward pair probability functions where the first argument refers to an occupied site of the preferred sublattice, the second ones to the subsequent and preceding sites, respectively. It is underlined that the present distinction between $p_{f}(1,1)$ and $p_{b}(1,1)$ is reasonable in the ordered phase for $E \neq 0$.

The above mentioned equations are solved numerically for different temperatures and electric fields. The Kawasaki hopping rate is used to avoid the singularity that comes from the nonanalytic behavior of the Metropolis rate, namely the probability of jumping from site $j$ to $k$ is

$$
\Gamma(j \rightarrow k)=\frac{1}{1+\exp [\beta(\Delta H-E \Delta r)]},
$$

where $\Delta H$ is the energy difference between the final and initial states, $\Delta r$ is the displacement, and $\beta=1 / T$. The temperature dependence of the order parameter $x(T)$ is very similar to those found by Dickman [5], thus his main conclusions remain unchanged. Evidently, our method reproduces the results obtained by Dickman for $E=0$ and $E \geq 2$ where there is no difference between $p_{f}(1,1)$ and $p_{b}(1,1)$. The critical temperature is slightly decreased when taking the symmetry breaking into consideration for $0<E<2$.

In Fig. 1 the solid line illustrates the calculated difference between $p_{f}(1,1)$ and $p_{b}(1,1)$ as a function of reduced temperature $T / T_{c}\left(T_{c}\right.$ means the equilibrium critical temperature) for a fixed electric field $E=0.4$. This difference is strongly related to the existence of the order parameter $x$; therefore, it disappears for high temperatures. Our calculation shows that this symmetry breaking is suppressed by the ordering process at low temperatures.

Monte Carlo simulations have been carried out to check the prediction of the present dynamical mean-field approximation. Using the usual techniques [9] the runs were performed on a half-filled lattice with periodic boundary conditions for different sizes $(L=40,64,128,256)$. Each run was started from an ordered state. After a thermalization of 2000 Monte Carlo steps per particle (MCS) we determined $p_{f}(1,1)$ and $p_{b}(1,1)$ by averaging over $10^{4}$ MCS. To decrease the statistical error we repeated this procedure 10 times at a given temperature. By this means we could avoid the uncertainty that comes from averaging in a polydomain structure which may appear for long time simulations below $T_{c}$. Rigorous analysis of

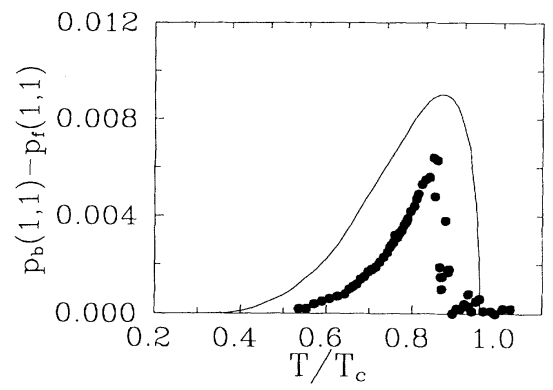

FIG. 1. Difference between $p_{b}(1,1)$ and $p_{f}(1,1)$ as a function of reduced temperature at a fixed electric field $E=0.4$. The solid line illustrates the theoretical prediction, the bullets represent Monte Carlo data.

the formation of the polydomain structure goes beyond the scope of the present paper [10].

Figure 1 demonstrates that the Monte Carlo data show qualitative agreement with our theoretical calculation in the single domain state. The size dependence seems to be irrelevant when measuring the above mentioned pair distribution functions.

Several simulations have been performed to visualize the typical elementary processes below $T_{c}$. Each particle residing in an ordered surrounding can jump and may return to the original site at low temperatures. In other words, one can observe particle-hole (Frenkel) pairs with a finite lifetime. The contribution of particle-hole pairs to $p_{f}(1,1)$ and $p_{b}(1,1)$ depends on their relative orientation. According to Eq. (1) the probability of forward (backward) jumps is greater (less) than that of the transverse ones. Consequently, the particle-hole pairs are "polarized" and give different contributions to $p_{f}(1,1)$ and $p_{b}(1,1)$.

Theoretical descriptions including the present one assume the existence of an ordered structure with infinite range of order. The breaking of forward-backward symmetry, however, is independent of the presence of longrange order. On the analogy of the one-dimensional system [6] this symmetry breaking is also observable for high temperatures, i.e., this phenomenon is not related to the phase transition. This fact is demonstrated by Monte Carlo simulations in Fig. 2 where the difference between the probability of the four-point configurations (1100) and (0011) on four subsequent sites along the field is plot-

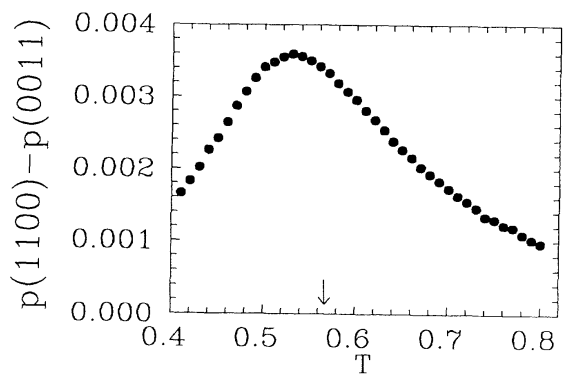

FIG. 2. Difference between the probability of four-point configurations (1100) and (0011) vs temperature for $E=0.4$. The arrow indicates the equilibrium critical temperature $T_{c}$. 
ted as a function of temperature for a fixed electric field $E=0.4$. The simulations were carried out on a $200 \times 200$ lattice by averaging over 50000 MCS. As expected, this type of symmetry breaking is suppressed by the ordering process at low temperatures and by the thermal fluctuations at high temperatures. It is emphasized that the same difference is found between other pairs of four-point configurations [i.e., (0101) and (1010)], in agreement with the one-dimensional results [6].

In summary, we have studied the forward and backward symmetry breaking in a half-filled two-dimensional lattice-gas model with a repulsive nearest-neighbor interaction. In this system the symmetry breaking may be investigated by introducing two pair probability functions, $p_{f}(1,1)$ and $p_{b}(1,1)$, along the driving field in the ordered (single-domain) state. These quantities were analyzed by using Monte Carlo simulations and by the modification of the dynamical mean-field theory within the framework of pair approximation. In the single-domain state the difference between $p_{f}(1,1)$ and $p_{b}(1,1)$ depends on the electric field, its value is strongly related to the order parameter, and it is suppressed by the ordering process in the low-temperature limit. The breaking of forward-backward symmetry, however, is observable between other $k$-point $(k \geq 4)$ configurations in agreement with the one-dimensional calculations. Monte Carlo simulations show that the symmetry breaking itself exists in this driven system independently of the presence of longrange order. The value characteristic of this symmetry breaking depends on $E$ and has a maximum below the equilibrium critical temperature.

It is expected that forward-backward symmetry breaking is characteristic of all driven lattice gases and it should be taken into consideration when rigorously analyzing the ordering process.

The authors thank Professor Ole G. Mouritsen and Thomas Hønger for their critical reading of the manuscript. This research was supported in part by the Hungarian National Science Fund (OTKA) under Grant No. T-4012.
* Permanent address: KFKI-Research Institute for Materials Science, H-1525 Budapest, P. O. Box 49, Hungary.

[1] B. Schmittmann, Int. J. Mod. Phys. B 4, 2269 (1990).

[2] S. Katz, J. L. Lebowitz, and H. Spohn, Phys. Rev. B 28, 1655 (1983); J. Stat. Phys. 34, 497 (1984).

[3] M. Q. Zhang, J.-S. Wang, J. L. Lebowitz, and J. L. Vallés, J. Stat. Phys. 52, 1461 (1988).

[4] K.-T. Leung, B. Schmittmann, and R. K. P. Zia, Phys. Rev. Lett. 62, 1772 (1989).

[5] R. Dickman, Phys. Rev. A 41, 2192 (1990).
[6] G. Szabó, A. Szolnoki, and L. Bodócs Phys. Rev. A 44, 6375 (1991).

[7] L. Onsager, Phys. Rev. 65, 117 (1944).

[8] R. Dickman, Phys. Rev. A 38, 2588 (1988).

[9] See, e.g., K. Binder and D. W. Heermann, Monte Carlo Simulation in Statistical Physics: An Introduction (Springer-Verlag, Berlin, 1988).

[10] The polydomain structure as a consequence of enhanced particle transport along the domain wall will be the subject of a subsequent paper. 